\title{
Thoracic Unilateral Spinal Cord Syndrome Following Neuraxial Anaesthesia for Periprosthetic Knee Fracture: A Case Report
}

\section{Nahir Chalup Torija1, Álvaro Ramiro Ruiz ${ }^{*}{ }^{\circledR}$, Rubén Rodriguez Salas ${ }^{1}$, Beatriz Fuentes Benito², Marina Nevado Villafruela ${ }^{3}$, Manuel Cortés Guerrero ${ }^{1}$}

\author{
${ }^{1}$ Anaesthesia, Critical Care Department and Pain Medicine, Hospital Universitario 12 de Octubre, Madrid, Spain \\ ${ }^{2}$ Hospital Universitario Fundación Alcorcón, Alcorcón, Spain \\ ${ }^{3}$ Centro de Salud de Torrelodones, Áreanoroeste, Torrelodones, Spain \\ Email: ^alraru86@gmail.com
}

How to cite this paper: Torija, N.C., Ramiro Ruiz, Á., Salas, R.R., Benito, B.F., Villafruela, M.N. and Guerrero, M.C. (2019) Thoracic Unilateral Spinal Cord Syndrome Following Neuraxial Anaesthesia for Periprosthetic Knee Fracture: A Case Report. Open Journal of Anesthesiology, 9 , 197-202.

https://doi.org/10.4236/ojanes.2019.911019

Received: February 12, 2019

Accepted: October 29, 2019

Published: November 1, 2019

Copyright $\odot 2019$ by author(s) and Scientific Research Publishing Inc. This work is licensed under the Creative Commons Attribution International License (CC BY 4.0).

http://creativecommons.org/licenses/by/4.0/

\section{(c) (i) Open Access}

\begin{abstract}
Neuraxial anaesthesia is widely used in surgical procedures; overall, epidural and intrathecal techniques. Nevertheless, several outcomes should be considered. The incidence of neurologic complications after neuraxial anaesthesia is not perfectly clear $(0 \%-0.08 \%)$, although there are several described cases of spinal cord ischemia. We present a case of thoracic unilateral spinal cord syndrome following lumbar spinal anaesthesia for periprosthetic knee fracture. Our patient suffered monoparesis in her left lower limb as well as decreasing of muscle strength and loss of tendon reflexes. The MNR showed left hyperintense intra-cord images from T7 to T12 attributed to spinal cord oedema and a lineal hypointensity related to minimal haematic component. What made this case surprising was the fact that spinal anaesthesia was performed between L3 and L4 and the patient did not suffer paraesthesia associated with local anaesthetic injection. She was treated with glucocorticoids, gabapentin and amitriptyline. She also was checked by physical rehabilitators, neurologists and Pain Unit physicians. We have found another case reported in the literature about thoracic cord injury after lumbar spinal puncture. In this paper, we report possible aetiologies according to a review and neurological evolution of the patient seven months later.
\end{abstract}

\section{Keywords}

Neuraxial Anaesthesia, Regional Anaesthesia, Neurologic, Complications, Spinal Cord Injury 


\section{Introduction}

If there is no contraindication, neuraxial techniques conform to the anaesthetic management of this patient. Its complications range from low entity (insignificant) to life threatening [1] [2] [3].

The pathophysiology of spinal cord injury associated with regional anaesthetic techniques has been reviewed in depth, including that related to mechanical trauma from direct needle/catheter injury or mass lesions, spinal cord ischemia or vascular injury from direct needle/catheter trauma, and neurotoxicity from local anaesthetics, adjuvants, or antiseptics. Specific recommendations are offered that may reduce the likelihood of spinal cord injury associated with regional anaesthetic or interventional pain medicine techniques [4].

\section{Case Report}

A 69-year-old woman (weight: $80 \mathrm{~kg}$; height $170 \mathrm{~cm}$ ) was admitted to the hospital for right periprosthetic knee fracture osteosynthesis. Her comorbidities were gastroesophageal reflux, breast cancer and primary knee arthroplasty surgery in 2014. Midazolam $2 \mathrm{mg}$ for premedication and cefazolin $2 \mathrm{~g}$ for antibiotic prophylaxis were administered. Ecoguided femoral nerve block was performed helped by neurostimulation and $20 \mathrm{ml}$ of levobupivacaine $0.25 \%$ was injected with no incidences. Then, intrathecal puncture was difficult and required three attempts. A forced position was necessary for a long time in lateral decubitus. Finally, an L3 - L4 spinal anaesthesia was performed and clear cerebrospinal fluid reflowed through Withacre $25 \mathrm{G}$ needle so $13 \mathrm{mg}$ of isobaric bupivacaine was administrated. During the technique; the patient presented self-limited paraesthesia not related to local anaesthetic injection. Osteosynthesis was performed in $85 \mathrm{~min}$; no complications were recorded. Her blood pressure and heart rate remained stable. $1250 \mathrm{ml}$ of crystalloid and $500 \mathrm{ml}$ of colloid solution were administrated. Transfusion of blood products was not required. The patient was transferred to the Recovery Unit. At the fourth hour of the immediate postoperative period, motor block was reversed in the broken leg but not in the healthy one. Two hours later, this condition remained with the only improvement she was able to extend her feet. Magnetic nuclear resonance (MNR) was ordered so as to rule out epidural haematoma (EH). The MNR showed left hyperintense intra-cord images from T7 to T12 attributed to spinal cord oedema and a lineal hypointensity related to minimal haematic component (Figure 1).

EH was discarded so urgent surgical treatment was dismissed by Neurosurgery Department. Dexamethasone $10 \mathrm{mg}$ following $8 \mathrm{mg}$ each 8 hours was started associated with amitriptyline and gabaphentin so as to avoid neuropathic pain. The patient was followed by our Neurology department and the pain Unit. The day after, a formal neurologic examination was performed with no deficits in her mental status nor cranial nerves. Motor response and sensitivity were preserved in upper limbs and in her fractured leg. Monoparesis in left lower limb was evidenced in the neurologic exam as well as decrease of muscle strength and 
loss of tendon reflexes. Besides, the patient presented absence of pain and temperature perception except for the dermatomes supplied by sacral nerve roots.

A week later a new MNR was performed showing less intra-cord hyperintensity (Figure 2). Neurologic examination on 25th day after the surgery was performed. Her left lower limb monoparesis remained. She presented pain and temperature sensitivity preserved in her foot but she kept diminished muscle strength and loss of tendon reflexes. With the diagnosis of unilateral anterior spinal cord syndrome the patient was discharged from hospital and she was admitted into a rehabilitation centre. Seven months later, the patient has recovered motor function but complete sensitive disorder remains. Nowadays she is able to stand up and she is walking with crutches. She does not suffer fecal incontinence but she needs bladder catheterization.

\section{Discussion}

Neuraxial anaesthesia is the most commonly used anaesthesia technique for total hip and knee replacement surgery; neuraxial anaesthesia has been associated with lower rate of admission to critical care services, a reduction of surgical site

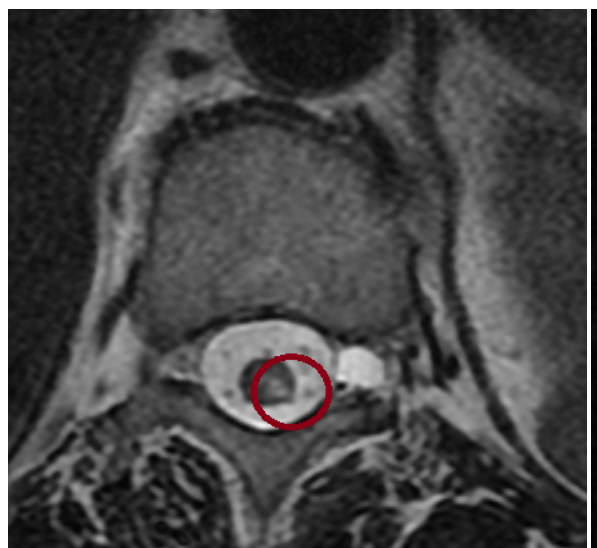

(a)

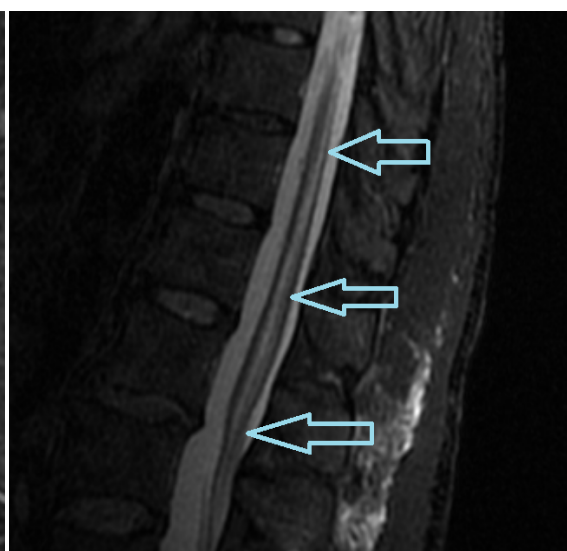

(b)

Figure 1. First MNR showing left hyperintense intra-cord images from T7 to T12 attributed to spinal cord oedema (red circle in (a) and blue arrows in (b)).
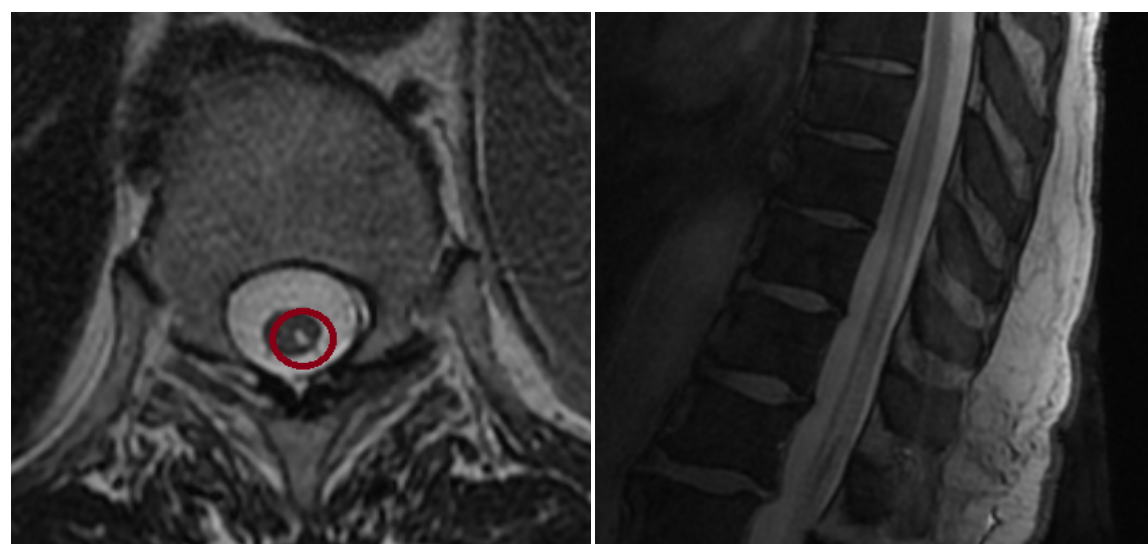

Figure 2. MNR a week later showing less intra-cord hyperintensity. 
infections, less incidence of deep venous thrombosis and a reduction in blood loss compared to general anaesthesia; decreased 30-day morbidity is not clear though [5] [6] [7] [8].

The incidence of postoperative neurological complications after neuraxial anaesthesia stands between $0 \%-0.08 \%$ [1]. Carpenter et al. performed a study in which they analyzed the incidence of hypotension (33\%), bradycardia (13\%), nausea $(18 \%)$, vomiting (7\%) and dysrhythmia (2\%). Their results indicated that these side effects may be reduced by minimizing peak block height (not above T5), using plain solutions of local anaesthetics and performing spinal puncture at or below L3 - L4 [3].

In our case, after ruling out epidural haematoma, we included as differential diagnosis: 1) spinal cord ischemia, although there was no description of hypotension during surgery and nor other symptoms were related. Besides the injury was located mainly at the posterior portion of the spinal cord; 2) traumatic aetiology, which is improbable as the puncture was performed at L3 - L4; 3) anaesthetic agent toxicity, but there was used an isobaric solution, being less harmful than hyperbaric ones.

A. Ramirez Bermejo et al. describe several cases analyzing possible risk factors and treatments for neuraxial anaesthesia complications, developing a therapeutic protocol. They include eight cases of neuraxial anaesthesia (spinal and epidural) for different surgical procedures; all of them describe paresthesia during the technique and had some type of damage in image tests (MNR). Those who followed a complete treatment had a better recovery than those not being treated. This protocol they applied included methylprednisolone, anti-inflammatory agents, anticonvulsant drugs and tricyclic antidepressants [2].

Our patient's treatment included dexamethasone in a glucocorticoid dose, gabapentin and amitriptyline. She also was checked and treated by physical rehabilitators, neurologists and doctors from the pain unit.

Costa Fabio also reported a case of a patient having left lower limb paresis without losing sensitivity, after spinal anaesthesia for hip replacement surgery. This patient's MNR showed a left cord hypointense image at T8 - T11 level concordant to ischemia. Nevertheless the cause remained unclear, it may have been produced by many different factors: intraoperative hypotension, anatomical variations, tranexamic acid use, local anaesthetics toxicity, etc. [1].

Another possible aetiology that must be taken into account is anterior spinal artery syndrome. The anterior spinal artery is supplied mainly by the Adamkiewicz artery, as well as multiple arterial branches. If this artery is occluded, there will be ischemia of the lower two-thirds of the spinal cord. Although there is wide variability in the spinal cord blood supply, we may observe unilateral muscle paralysis with unilateral spinal cord ischemia due to this artery occlusion [1].

We followed the practice advisory on neurologic complications associated to regional anaesthesia and pain medicine published by the America Society of Regional Anaesthesia and Pain Medicine (ASRA) [9], to manage this situation. 


\section{Conclusions}

Severe neurological complications due to neuraxial anaesthesia are not frequent.

Aetiology in these cases is not usually clear and it usually has multifactorial causes (patient characteristics, body position during surgery, spinal cord blood supply, and hemodynamic changes).

Early diagnosis is crucial to start the treatment. It is important to consider all possible contributing factors, avoiding focusing on just one cause.

Multidisciplinary management of the patient is essential to get the best outcomes, overall rehabilitation treatment and long term follow-up. So as to manage neurologic complications associated with regional anaesthesia soon and better we consider as a priority to adapt ASRA's practice advisory to our Centre.

\section{Conflicts of Interest}

The authors declare no conflicts of interest regarding the publication of this paper.

\section{References}

[1] Fabio, C., Romualdo, D.B., Eugenio, A.F., Vittoradolfo, T., Massimiliano, V.A. and Giovanna, R. (2017) Thoracic Unilateral Spinal Cord Injury after Spinal Anaesthesia for Total Hip Replacement: Fate or Mistake? Turkish Journal of Anaesthesiology Reanimation, 45, 116-118. https://doi.org/10.5152/TJAR.2016.32967

[2] Bermejo, A.R., Aldrete, J.A., Cubillo, N.C.G., Sánchez, S.B., Ghaly, R. and Rank, A.K. (2009) Défi cits neurológicos y aracnoiditis secundarios a anestesia neuroaxial: rol de parestesias, punciones durales, anestésicos locales y género. Protocolo para su tratamiento. Revista de la Sociedad Española del Dolor, 16, 330-343. https://doi.org/10.1016/S1134-8046(09)72541-8

[3] Carpenter, R.L., Caplan, R.A., Brown, D.L., Stephenson, C. and Wu, R. (1992) Incidence and Risk Factors for Side Effects of Spinal Anesthesia. Anesthesiology, 76, 906-916. https://doi.org/10.1097/00000542-199206000-00006

[4] Neal, J.M., Kopp, S.L., Pasternak, J.J., Lanier, W.L. and Rathmell, J.P. (2015) Anatomy and Pathophysiology of Spinal Cord Injury Associated With Regional Anesthesia and Pain Medicine: 2015 Update. Regional Anesthesia \& Pain Medicine, 40, 506-525. https://doi.org/10.1097/AAP.0000000000000297

[5] Perlas, A., Chan, V.W. and Beattie, S. (2016) Anesthesia Technique and Mortality after Total Hip or Knee Arthroplasty: A Retrospective, Propensity Score-Matched Cohort Study. Anesthesiology, 125, 724-731. https://doi.org/10.1097/ALN.0000000000001248

[6] Smith, L.M., Cozowicz, C., Uda, Y., Memtsoudis, S.G. and Barrington, M.J. (2017) Neuraxial and Combined Neuraxial/General Anesthesia Compared to General Anesthesia for Major Truncal and Lower Limb Surgery: A Systematic Review and Meta-Analysis. Anesthesia \& Analgesia, 125, 1931-1945. https://doi.org/10.1213/ANE.0000000000002069

[7] Rodgers, A., Walker, N., Schug, S., McKee, A., Kehlet, H., van Zundert, A., Sage, D., Futter, M., Saville, G., Clark, T. and MacMahon, S. (2000) Reduction of Postoperative Mortality and Morbidity with Epidural or Spinal Anaesthesia: Results from Overview of Randomised Trials. BM), 321, 1493.

https://doi.org/10.1136/bmj.321.7275.1493 
[8] Johnson, R.L., Kopp, S.L., Burkle, C.M., Duncan, C.M., Jacob, A.K., Erwin, P.J., Murad, M.H. and Mantilla, C.B. (2016) Neuraxial vs. General Anaesthesia for Total Hip and Total Knee Arthroplasty: A Systematic Review of Comparative-Effectiveness Research. British Journal of Anaesthesia, 116, 163-176.

https://doi.org/10.1093/bja/aev455

[9] Neal, J.M., Bernards, C.M., Hadzic, A., et al. (2008) ASRA Practice Advisory on Neurologic Complications in Regional Anesthesia and Pain Medicine. Regional Anesthesia \& Pain Medicine, 33, 404-415.

https://doi.org/10.1097/00115550-200809000-00003 